\title{
RELEVAMIENTO DE ESCARABEIDOS (INSECTA, COLEOPTERA) EN PASTIZAL NATURAL, PASTO LLORÓN Y MAIZ, EN EL ESTE DE LA PROVINCIA DE SAN LUIS (ARGENTINA)
}

\author{
Bonivardo, S. L. ${ }^{1 ;}$ Martinez, A. N. ${ }^{1}$; \\ Perassi Mana, $G .{ }^{1}$ \& Suarez, A. \\ RESUMEN
}

Los escarabeidos son importantes integrantes de la fauna edafícola pero pueden convertirse en plaga en suelos con siembra directa y en aquellos poco perturbados. Con el fin de determinar las especies de Scarabaeidae y su abundancia en tres condiciones, suelo cultivado (maíz), pasto llorón implantado y pastizal natural, en la Región Fitogeográfica I de la provincia de San Luis (Argentina), se realizaron muestreos completamente aleatorizados, durante dos períodos. En cada condición se tomaron tres muestras de suelo de $1 / 8 \mathrm{de}^{2}$ a $30 \mathrm{~cm}$ de profundidad. Las larvas de escarabeidos se determinaron a nivel de especie según claves específicas, observándose ocho especies. Se determinaron en todas las condiciones de disturbio de suelo: Cyclocephala modesta Burmeister, Cyclocephala putrida Burmeister y Anomala testaceipennis Blanchard, siendo las dos primeras las más abundantes; Lyogenis sp. Guérin-Méneville y Philochloenia bonariensis Blanchard se observaron sólo en pastizal natural, Cyclocephala signaticollis Burmeister sólo en pasto llorón, Bothinus striatellus Hope estuvo presente sólo en maíz y Leucothyreus sp. MacLeay se presentó en pasto llorón y maíz.

Palabras Claves: Diversidad, gusanos blancos, disturbio de suelo.

\begin{abstract}
Scarabaeidae survey of natural pastures, weeping lovegrass and corn in eastern province of San Luis (Argentina).

The scarabs beetle important as members of the edaficola wildlife, can be a pest in soil tillage and those little disturbed. In order to determine the species of Scarabaeidae and abundance in three conditions, cultivated land (maize), implanted weeping lovegrass and natural pasture in Phytogeographical Region I of the province of San Luis (Argentina), samples were taken completely randomized, for two periods. In each condition three soil samples $1 / 8 \mathrm{~m} 2$ to $30 \mathrm{~cm}$ deep were taken. Scarabaeidae larvae were determined to species level according to specific keys, eight species observed. They were determined in all conditions of soil disturbance: Cyclocephala modesta, Cyclo-
\end{abstract}

1.- Proyecto C y T - UNSL No 140512 Dpto. Cs. Agropecuarias. Facultad de Ingeniería y Cs. Agropecuarias (FICA). UNSL. Av. 25 de Mayo 384. Villa Mercedes, provincia de San Luis. Email: sbonivardo@gmail.com Manuscrito recibido el 27 de julio de 2015 y aceptado para su publicación el 5 de abril de 2016. 


\section{S. L. Bonivardo et al.}

cephala putrida and Anomala testaceipennis, the former being the most abundant two; Lyogenis sp. and Philochloenia bonariensis were seen only in natural grassland, Cyclocephala signaticollis only weeping lovegrass, Bothinus striatellus was present only in corn and Leucothyreus sp. he was presented in weeping lovegrass and corn.

Key words: diversity, white worms, soil disturbance.

\section{INTRODUCCION}

En la provincia de San Luis, la tendencia a optimizar el recurso forrajero para atender la demanda producida por el ganado bovino, fue reorganizada dentro de los sectores donde la siembra directa no ha avanzado debido a las condiciones edafoclimáticas. Entre las alternativas existentes, se destacan: el aprovechamiento del pastizal natural con usos estratégicos y la implantación de pasturas megatérmicas. El pasto llorón Eragrostis curvula (Schrader) Nees es una pastura perenne estival con cualidades adaptativas que se destaca en la región semiárida pampeana (5).

La familia Scarabaeidae (Insecta: Coleoptera) incluye importantes integrantes de la fauna edafícola conocidos en varios lugares del mundo por producir daños en las partes subterráneas de las plantas cultivadas. Unas 15.000 especies de este grupo tienen larvas edafícolas cuyos hábitos alimentarios aún no han sido precisados (10). A las larvas de las especies de escarabeidos rizófagas se las denomina comúnmente "complejo de gusanos blancos" del suelo. Detalles sobre la biología y el comportamiento de estas larvas han sido proporcionalmente poco estudiados (10).

El complejo de gusanos blancos es uno de los grupos de insectos más relacionados con la siembra directa, pues al no perturbarse el hábitat aumentaría la tasa neta de crecimiento de sus poblaciones a través de los años (8). Se señala que según las rotaciones de cultivos en sistemas de siembra directa, la población de gusanos blancos disminuye aproximadamente un 33\% cuando el antecesor fue soja en comparación al antecesor maíz, y se incrementa de tres a cuatro veces al pasar de labranza convencional a siembra directa y a su vez de ésta a las pasturas perennes (7). En Uruguay las especies que frecuentemente fueron encontradas en situaciones de siembra directa fueron: Diloboderus abderus Sturm, Cyclocephala signaticollis Burmeister, Cyclocephala modesta, Cyclocephala putrida y Heterogeniates bonariensis Ohaus. La primer especie tiene marcada preferencia para oviponer en suelos compactos y con presencia de paja; las larvas de C. signaticollis son encontradas en una gran variedad de situaciones (campo natural, praderas y cultivos de trigo, maíz, sorgo y girasol), observándosela como especie predominante en sistemas agrícolas en siembra directa; $C$. modesta y $H$. bonariensis están asociada a sistemas agrícolas cuando en la rotación se integran leguminosas forrajeras (13).

En distintos estudios realizados en la provincia de San Luis se cita la presencia de Diloboderus abderus, Anomala testaceipennis, Cyclocephala putrida, Cyclocephala modesta, Cyclocephala signaticollis, Liogenys sp., Leucothyreus sp., Bothinus sp. y Philochloenia bonariensis $(3,9)$. Con respecto a la diversidad de los escarabeidos asociados a pastizales, se de- 